\title{
Experimental Confirmation of the Doubts about Authenticity of Event GW150914
}

\author{
Alexander V. Lukanenkov \\ Moscow State Linguistic University, Moscow, Russia \\ Email: a_v_luk@mail.ru
}

How to cite this paper: Lukanenkov, A.V. (2017) Experimental Confirmation of the Doubts about Authenticity of Event GW150914. Journal of Applied Mathematics and Physics, 5, 538-550. https://doi.org/10.4236/jamp.2017.52046

Received: January 18, 2017

Accepted: February 25, 2017

Published: February 28, 2017

Copyright $\odot 2017$ by author and Scientific Research Publishing Inc. This work is licensed under the Creative Commons Attribution International License (CC BY 4.0).

http://creativecommons.org/licenses/by/4.0/ (c) (i) Open Access

\begin{abstract}
It was produced processing of the LIGO 14.09.2015 registration data. It was established that the chirp signals (signals of merger of black holes) are absent in the data. This is proved by using a coherent filtering and also two-stage causal filtering. Soliton-like signals such as wavelet "Mexican hat" are found by filtering based on the Butterworth filter. These signals are different in polarity, and their spectra are quite different. Doubts about the authenticity of the detection of chirp signals, which announced on February 11, 2016, are justified by the results the conducted analysis.
\end{abstract}

\section{Keywords}

Gravitational Waves, Optimal Detector, Linear Frequency Modulated Signal, Chirp Signal, Band Pass Filtering, Matched Filter, Optimal Signal Processing, Causal Filter

\section{Introduction}

In 1916, Albert Einstein predicted the existence of gravitational waves [1].

The discovery of the binary pulsar system PSR B1913 + 16 by Hulse and Taylor [2] and subsequent observations of its energy loss by Taylor and Weisberg

[3] demonstrated the existence of gravitational waves.

Experiments to detect gravitational waves began with Weber [4].

It was announced the observation of gravitational waves from merging black holes thanks to the two signals registered according to observatories LIGO Hanford $(H 1)$ and the LIGO Livingston $(L 1)$ in [5].

This paper presents the results of processing of registration data posted on the website LIGO (https://losc.ligo.org/events/GW150914) [6].

\section{Registration Data of Two Observatories}

The time of arrival of gravitational waves: 
$-T_{\text {detect }}=1,126,259,462.39=$ September 14, 2015, 09:50:45.39 UTC, respective signals detected in the frequency band $[35 \div 350 \mathrm{~Hz}]$, the events have a combined signal-to-noise ratio $\mathrm{SNR}_{\text {announced }} \approx 24$ [5] [6].

These data were presented in February-May, 2016 on the website [6], later, in July 2016 the time of the gravitational wave arrival was corrected and been removed 0.39 seconds, this is roughening of accuracy the time of arrival.

Because of this it is now the time of arrival of gravitational waves is specified without hundredths of a second on the website [6]:

$T_{\text {detect }}=1,126,259,462=$ September 14 2015, 09:50:45 UTC.

It was announced in [5] about the delay between the observatories which is $\approx$ $6.9 \mathrm{~ms}$ (arrived first at $L 1$ and $6.9 \mathrm{~ms}$ later at $H 1$ ).

It is strange that removed hundredths of a second, no time of signal arrivals at the $L 1$ and $H 1$, but announced about the delay between the arrival of the GWwave at different observatories to within a few thousandths of a second.

Time series (fragments) strains $h_{L}(t), h_{H}(t)$ are contained in the files:

L-L1_LOSC_4_V1-1126259446-32.txt, H-H1_LOSC_4_V1-1126259446-32.txt.

The corresponding random processes have a bandwidth $[10 \div 2048 \mathrm{~Hz}]$.

Fragments $h_{L}(t), h_{H}(t)$ have a duration of 32 seconds, strain time series centered at

GPS $1126259462=$ September 14 2015, 09:50:45 UTC.

Sampling frequency $f_{d}=4096 \mathrm{~Hz}$.

Start Time of the fragment $T_{\text {start }}=1,126,259,446=$ September 14 2015, 09:50:29

UTC, it is 16 seconds before the time of signal arrival $T_{\text {detect }}=16.39 \mathrm{sec}$.

Further seconds and its share are relative $T_{0}=09: 50: 45$ or $T_{\text {start } 0}=09: 50: 29$.

On the page "Data release for event GW150914"

(https://losc.ligo.org/events/GW150914/) are given:

-results of processing carried out by the members of LIGO Collaboration (Figure 1);

-signals $s_{H}(t), s_{L}(t)$ (Figure 1) according to observation 14.09.2015.

Experimental templates, shown in Figure 1, can be described as a first approximation by the relation:

$$
S(t)=A(t) \cos \left(\varphi_{0}+2 \pi\left(f_{0} t+\frac{\beta t^{2}}{2}\right)\right)
$$

where $A(t)$-the signal amplitude;

$f_{0}$-the carrier frequency;

$\varphi_{0}$-the initial phase;

$\beta$-the speed of change of frequency;

$f_{0}=\left(F_{\text {max }}+F_{\text {min }}\right) / 2$;

$\beta=\left(F_{\max }-F_{\min }\right) / T \mathrm{~s}$;

$F_{\max }, F_{\min }-$ minimum and maximum value of the signal frequency;

Ts-signal duration.

These signals are called linear frequency modulated (LFM) signals. 

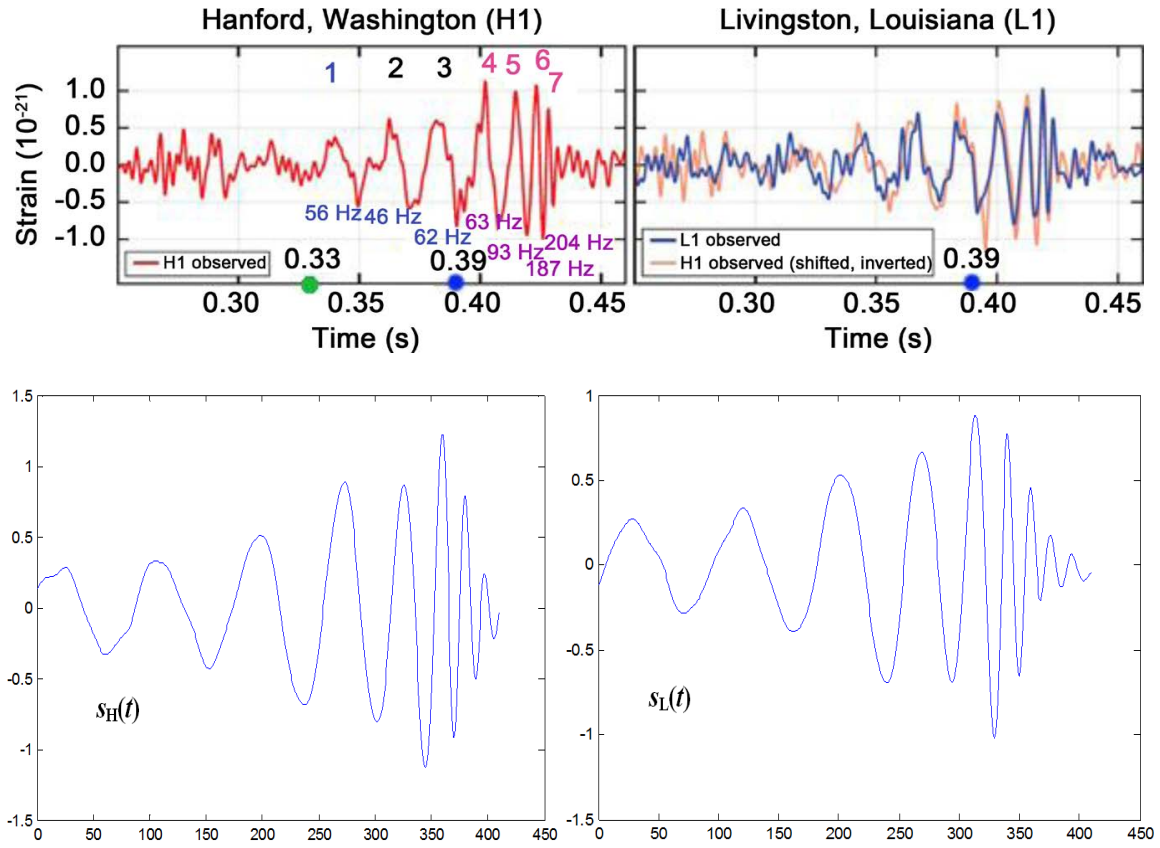

Figure 1. Top part. The gravitational-wave event GW150914 observed by the LIGO Hanford ( $H 1$, left column panels) and Livingston ( $L 1$, right column panels) detectors. Times are shown relative to September 14, 2015 at 09:50:45 UTC. For visualization, all time series are filtered with a $35-350 \mathrm{~Hz}$ band-pass filter to suppress large fluctuations outside the detectors' most sensitive frequency band, and band-reject filters to remove the strong instrumental spectral lines [5]. Declared time of the entry signal (0.39 s) indicated by blue circles [5]. Numbers and frequencies of wave phases (left): The true signal entry into $0.33 \mathrm{~s}$ (green circle). Bottom part: Experimental templates on observatories $s_{H}(t)$ (Hanford), $s_{L}(t)$ (Livingston) [6]. The horizontal axis-number in the data array, on the ordinate axis scaling factor $k=10^{-21}$ is sometimes omitted.

\section{Invariants of Signals before and after Filtering}

1) Linear-frequency modulated signal (LFM-signal), LFM-signal = Chirp signal. Chirp signal stays the chirp signal after passing through the band filter.

\begin{tabular}{|c|c|c|}
\hline $\begin{array}{c}\text { LFM-signal } \\
{\left[\mathrm{F}_{\text {min }}, \mathrm{F}_{\text {max }}\right]} \\
X(t)\end{array}$ & $\rightarrow$ & $\begin{array}{c}\text { LFM-signal } \\
{\left[\mathrm{f}_{\mathrm{n}}, \mathrm{F}_{\text {max }}\right]} \\
Y(t) \\
\mathrm{F}_{\text {min }}<\mathrm{f}_{\mathrm{n}}<\mathrm{F}_{\text {max }}, \mathrm{f}_{\mathrm{b}}>\mathrm{F}_{\text {max }}\end{array}$ \\
\end{tabular}

Filtering is defined by the convolution:

$$
Y(t)=X(t) \otimes h_{f}(t),
$$

where $h_{f}(t)$-impulse response of a filter;

$X(t)$-input process;

$Y(t)$ - the filtered process;

$\otimes$ is the sign of the convolution.

2) The integral of the square of the signal remains practically unchanged if the signal in the filter band. This is a consequence of Parseval's theorem.

$$
F_{Y}(f)=F_{X}(f) \cdot H(f) .
$$




$$
\begin{aligned}
& |H(f)| \approx 1 \Rightarrow \int_{t-T / 2}^{t+T / 2} X(\tau)^{2} \mathrm{~d} \tau \approx \int_{F_{\max }-\Delta f}^{F_{\max }+\Delta f}\left|F_{X}(f)\right|^{2} \cdot 2 \mathrm{~d} f \\
& \approx \int_{F_{\max }-\Delta f}^{F_{\max }+\Delta f}\left|F_{Y}(f)\right|^{2} \cdot 2 \mathrm{~d} f \approx \int_{t-T / 2}^{t+T / 2} Y(\tau)^{2} \mathrm{~d} \tau,
\end{aligned}
$$

where $H(f)$-the transfer function of the filter, $H(f)=F\left(h_{f}\right)$-a spectrum filter's impulse response, $|H(f)|$-amplitude-frequency characteristic(AFC) of the filter.

3) The spectrum of the signal after filtering remains practically unchanged if the signal is filtered in band, $|H(f)| \approx 1$.

4) If the registered signals $S_{H}(t)$ and $S_{L}(t)$ and they coincide with the delay and inversion (Figure 1), as stated in [5] [6], their spectra

$$
\begin{aligned}
& \left(\left|F s_{H}(f)\right| \approx\left|F s_{L}(f)\right|\right) \text { are almost equal after filtering. } \\
& \quad s_{H}(t)=-s_{L}(t+\tau), \quad x_{L}(t)=s_{L}(t)+n_{L}(t), x_{H}(t)=s_{H}(t)+n_{H}(t), \\
& \quad s_{L f}(t)=s_{L}(t) \otimes h_{f}(t), s_{H}(t)=s_{H}(t) \otimes h_{f}(t), \\
& H(f)=F_{h_{f}}(f), F s_{L f}(f)=F s_{L}(f) \cdot H(f), F s_{H f}(f)=F s_{H}(f) \cdot H(f), \\
& |H(f)| \approx 1 \Rightarrow F s_{L f}(f) \approx-F s_{H f}(f) \Rightarrow\left|F s_{L f}(f)\right| \approx\left|F s_{H f}(f)\right|
\end{aligned}
$$

Amplitude spectra $\left(\left|F s_{H f}(f)\right| \approx\left|F s_{L f}(f)\right|\right)$ are almost equal after filtering in the filter band $(|H(f)| \approx 1)$.

\section{The Procedure for the Detection of Known Signals}

Detection of gravitational chirp signal is based on the selection of one from two alternative hypotheses:

$$
\begin{aligned}
& H_{0}: \begin{aligned}
x_{H}(t) & =n_{H}(t), \\
x_{L}(t) & =n_{L}(t),
\end{aligned} \\
& H_{1}: \begin{aligned}
x_{H}(t) & =n_{H}(t)+s_{H}(t),-G W \text {-wave chirp signal is present. } \\
x_{L}(t) & =n_{L}(t)+s_{L}(t),
\end{aligned}
\end{aligned}
$$

From the mathematical theory of statistics follows [7] that the optimal receiver represents matched filtering procedure and then comparing to a threshold.

The matched filter has an impulse response equal to the inverted templates (Figure 1):

$$
T_{L}(t)=s_{L}\left(T_{\text {template }}-t\right), \quad T_{H}(t)=s_{H}\left(T_{\text {template }}-t\right),
$$

$T_{\text {template }}$-template duration (duration of impulse response).

The matched filter of input records in the band $[35 \div 350 \mathrm{~Hz}]$ with the signals, represented in Figure 1, is written by the relations:

$$
h_{1 f}(t)=h_{L f}(t) \otimes T_{L}(t), h_{2 f}(t)=h_{H f}(t) \otimes T_{H}(t),
$$

where $h_{L f}(t)=h_{L}(t) \otimes h_{f}(t), h_{L f}(t)=h_{H}(t) \otimes h_{f}(t)$-filtered processes after bandpass filtering in the band $[35 \div 350 \mathrm{~Hz}]$.

The procedure for testing the hypothesis of the presence of chirp signals in the records: 
1) Produced filtering $h_{H}(t)$ and $h_{L}(t)$ and find $h_{H f}(t)$ and $h_{L f}(t)$.

2) The convolution is produced in accordance with (Equation (7)) and find $h_{1 f}(t)$ and $h_{2 f}(t)$.

3) Modules $h_{1 f}(t)$ and $h_{2 f}(t)$ are compared with thresholds $\left(P_{1}, P_{2}\right)$.

$$
\begin{aligned}
& \left|h_{1 f}(t)\right|>P_{1} \Rightarrow \text { true } H_{1} \text { else } H_{0} \\
& \left|h_{2 f}(t)\right|>P_{2} \Rightarrow \text { true } H_{1} \text { else } H_{0}
\end{aligned}
$$

The signal/noise ratio is determined by the formula:

$$
\mathrm{SNR}=A_{\max } / \sigma_{n} .
$$

The results of the matched filter in accordance with the Equations (7) are shown in Figure 2, the signal/noise ratio $\mathrm{SNR}_{\text {real }} \approx 3$.

Such a small value of the $\operatorname{SNR}\left(\mathrm{SNR}_{\text {real }} \ll \mathrm{SNR}_{\text {announced }}=24\right)$ leads to the inevitable conclusion about doubtfulness of the detected signals, which are stated in [5] [6].

For example, a mixtures of signal and noise were formed to demonstrate the matched filtering efficiency:

$$
\begin{aligned}
& x_{L}(t)=h_{L}(t) \otimes h_{f}(t)+s_{L}\left(t-\tau_{s}\right) \frac{\sigma_{L}}{\sigma_{s}} k_{\mathrm{SNR}}, \\
& x_{H}(t)=h_{H}(t) \otimes h_{f}(t)+s_{H}\left(t-\tau_{s}\right) \frac{\sigma_{H}}{\sigma_{S}} k_{\mathrm{SNR}},
\end{aligned}
$$

where $h_{f}(t)$-impulse response of a band filter $[35 \div 350 \mathrm{~Hz}]$;

$h_{L}(t), h_{H}(t)$-time series of strains;

$\sigma_{s}$-rms signal $s(t)$;

$\sigma_{L}\left(\sigma_{H}\right)$-rms noise $n_{L}(t)\left(n_{H}(t)\right)$;

$\sigma=\sqrt{\operatorname{Disp}(h(t))}, k_{\mathrm{SNR}}$-factor depending on SNR;

$\tau_{s}$ - time of signal arrival.

Mixtures of signal and noise $x_{L}(t), x_{H}(t)$ are calculated for the following parameters: time of model signal arrival $\tau_{s} \approx 15 \mathrm{sec}, k_{\mathrm{SNR}}=1.0$.

Matched filtering results $x_{L}(t), x_{H}(t)$ with model signals $s_{H}(t)$ and $s_{L}(t)$ shown in Figure 2, the lower part.

The signal/noise ratio at the output $\mathrm{SNR}_{\text {out }}$ is determined by the $\mathrm{SNR}_{\text {in }}$ and gain at the expense of processing $\left(B_{\mathrm{SNR}}\right)$ :

$$
\mathrm{SNR}_{\text {out }}=B_{\mathrm{SNR}} \cdot \mathrm{SNR}_{\mathrm{in}} \text {. }
$$

For formed processes $x_{L}(t), x_{H}(t)$ signal/noise ratio at the input in the simulation:

$$
\mathrm{SNR}_{\text {in mod }} \approx 2.323 \text {. }
$$

It is clearly distinguished model signals to the 15-th second after matched filtering on Figure $2, \mathrm{SNR}_{\text {out mod }} \approx 8.9-10.7$ when the input signal/noise ratio $\approx$ 2.323 .

Input signal/noise ratio for gravitational signals in Figure 1.

$$
\mathrm{SNR}_{\mathrm{inL}} \approx 5.5, \mathrm{SNR}_{\mathrm{inH}} \approx 5.25
$$

At such values $\mathrm{SNR}_{\text {in }}$ by the Equation (10) 

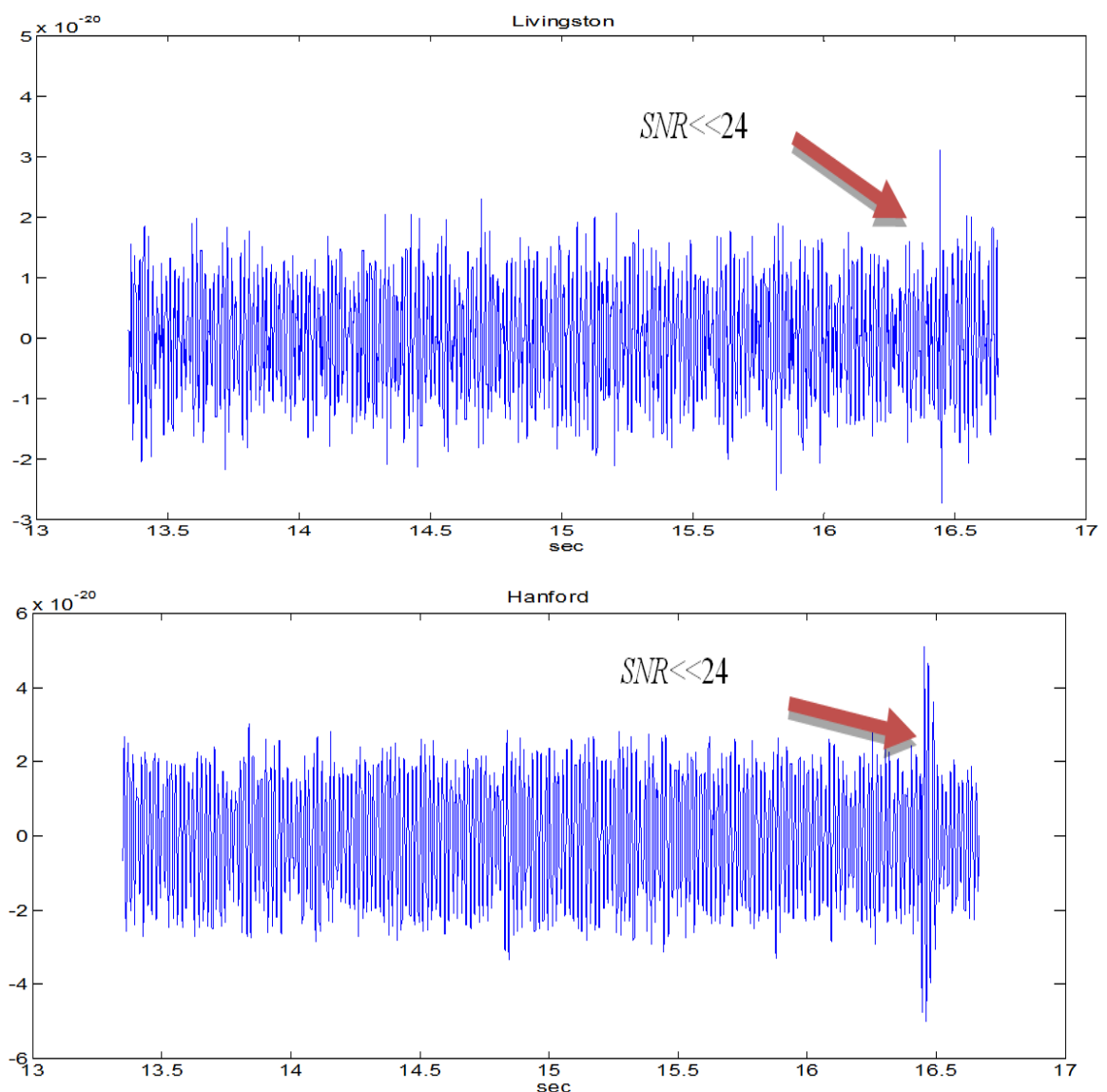

(a)
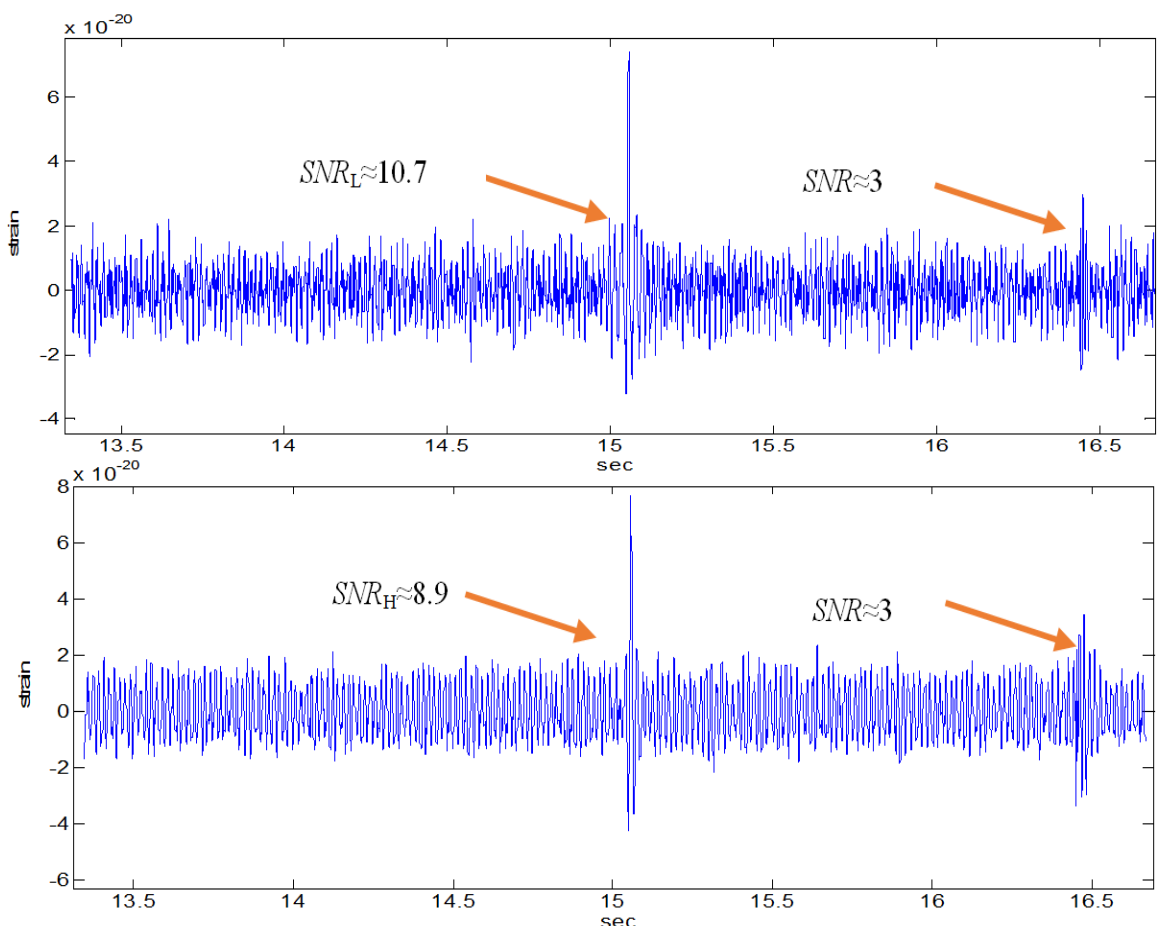

(b)

Figure 2. (a) The results of the matched filter $h_{L}(t), h_{H}(t)$; (b) The results matched filtering the mixture of signals and noises $x_{L}(t), x_{H}(t)$. 


$$
\mathrm{SNR}_{\text {out }}>8.9 * 5.25 / 2.323=20,
$$

that more than doubled when the model signals.

If the real signals are in Figure 1, after the matched filter would have $\mathrm{SNR}_{\text {out }}>$ 20, but it is not observed (Figure 2, top).

A small rise seen near 16-th second, corresponding $\mathrm{SNR}_{\text {real }} \approx 3$, as a result of matched filtering (the upper part of Figure 2), which is much smaller than it should be (Equation (11)).

Thus, the hypothesis $H 1$ is not confirmed.

This means: the chirp signals are absent in the original records (Figure 1).

\section{Definition of the Signals and Their Parameters}

If the chirp signals are absent in registration data (Figure 1), it is necessary to determine the type of signals that are in LIGO14.09.2015 records.

\subsection{Evaluation of the Times of Signal Arrivals}

For this purpose, the optimum detector is used, which calculates the likelihood ratio:

$$
L\left(h_{H} / s\right)=\ln \frac{P\left(h_{H} / s\right)}{P\left(h_{H} / n\right)}, L\left(h_{L} / s\right)=\ln \frac{P\left(h_{L} / s\right)}{P\left(h_{L} / n\right)} .
$$

From (Equation (12)) it follows that detection of the unknown signal is based on the computation of the functional in the first approximation [7] [8]:

$$
U(t)=\int_{f_{l}}^{f_{h}} \frac{G_{t+T}(f)}{G_{t-T}(f)} \mathrm{d} f,
$$

where $G_{t+T}(f), G_{t-T}(f)$-energy process spectra before and after the current time $t, f_{l}, f_{h}$-lower and upper frequency range of the signal.

Looking at Figure 3, you can see:

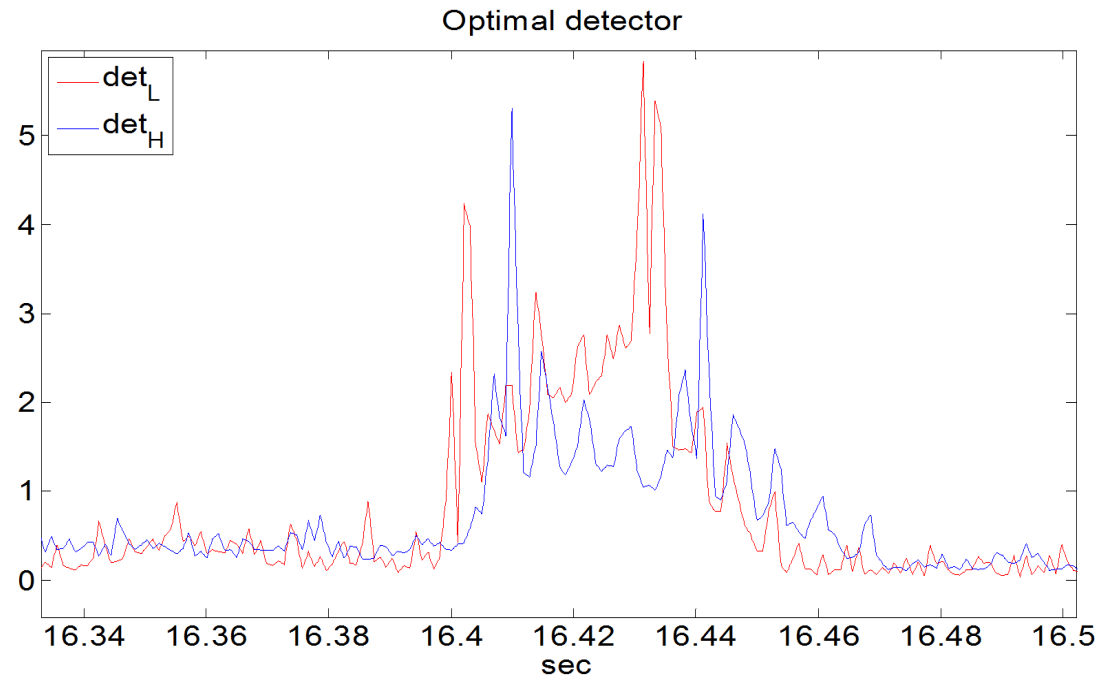

Figure 3. The results of the optimal detector Hanford $(H 1)$-blue line, Livingston $(L 1)$ red line. 
- Confident detection signals according to the observatory $L 1, H 1$;

- signal arrived first at $L 1$ and $\leq 10 \mathrm{~ms}$ later at $H 1$;

- $\mathrm{SNR} \geq 7$;

- arrival time at $L 1$ earlier $16.4 \mathrm{sec}$.

\subsection{Definition of Signal Waveforms Using Filtration}

This is done using a two-stage causal filtering:

1st step. Butterworth filter, $35-350 \mathrm{~Hz}$ band, the filter order $=8$.

2nd step. Butterworth filter, $60-450 \mathrm{~Hz}$ band, the filter order $=4$ (to notch components at frequencies near $32 \mathrm{~Hz}$ and $60 \mathrm{~Hz}$ ).

According to [5] [6], the amplitude of the wave phases №4 - $7 \quad A \approx 1 \times 10^{-21}$ (Figure 1), and their frequency is greater than $80 \mathrm{~Hz}$ and below $250 \mathrm{~Hz}$, so after a two-stage filtration $(\operatorname{AFC}|H(f)| \approx 1$ ), these wave phases have not practically change.

However, these wave phases are not observed in the time interval $16.39 \mathrm{sec}-$ $16.45 \mathrm{sec}$ in Figure 4, and there are pulse signals (amplitude signals from $1 \times 10^{-21}$ to $2.5 \times 10^{-21}$ ).

This apparent contradiction, and thereby violated №1 invariance property. Similarly, we can be sure of violating the invariance property №2.

In more detail the detected signals are represented in Figure 4 (Regime "Magnifier").

Signals (Figure 4, Regime "Magnifier") like wavelets "Mexican hat", "Sombrero".

They have the property of solitons:

the higher the frequency, the greater the amplitude,

$$
\max A_{L}>\max A_{H}, f_{L}>f_{H}
$$

Spectra of these signals are shown in Figure 5.

It follows from Figure 5:

$$
\begin{aligned}
& \max S_{H}(f) \approx 165 \mathrm{~Hz}, \max S_{L}(f) \approx 205 \mathrm{~Hz} ; \\
& \Delta F=F_{\max L}-F_{\max H} \approx 40 \mathrm{~Hz}=205-165 \mathrm{~Hz} .
\end{aligned}
$$

Spectrum of Signal $L 1$ is a higher frequency than spectrum of signal $H 1$ and invariants (№3, 4) are not performed.

This is a clear contradiction to the declared data about detected signals [5] [6], and contradicts the invariance properties of the spectra of signals at filtering (Invariants№№3, 4).

It means: $s_{H}(t) \neq-s_{L}(t+\tau)$ !

\section{Discussion of Processing Results}

Different types of filtration are used in detection of signal with a priori unknown form: bandpass, whitening(rectify), etc. [7] [8].

Physically realizable filter whitening is based on the use of optimal filtering Wiener-Kolmogorov theory [9] [10] and Levinson-Durbin procedures [11] [12].

In [5] [6] abandoned this approbated way of whitening of noises. 

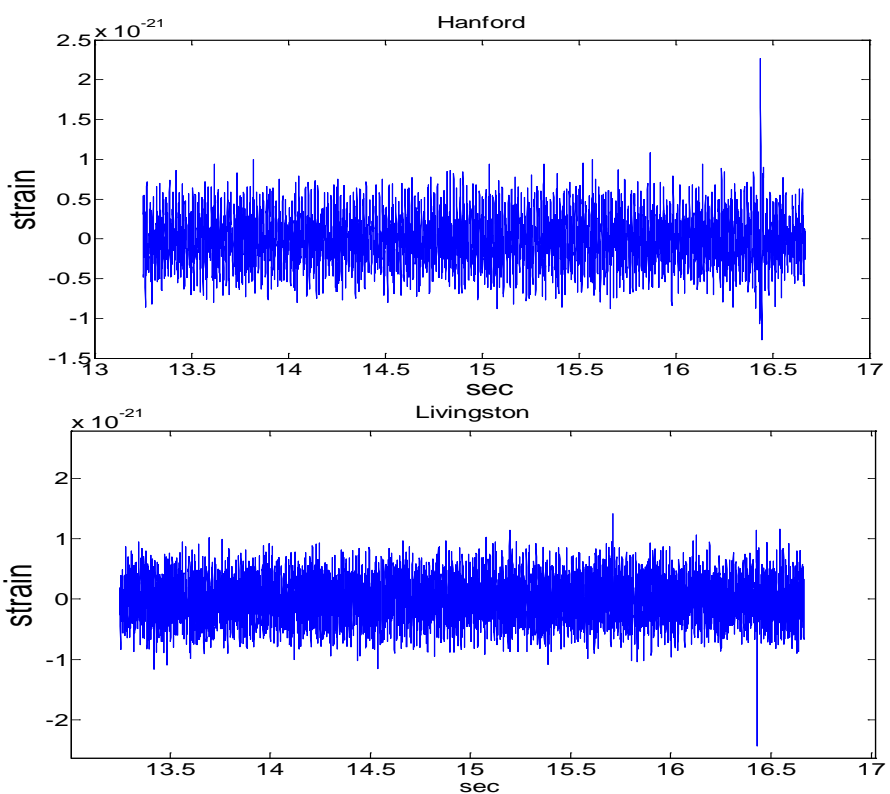

(a)
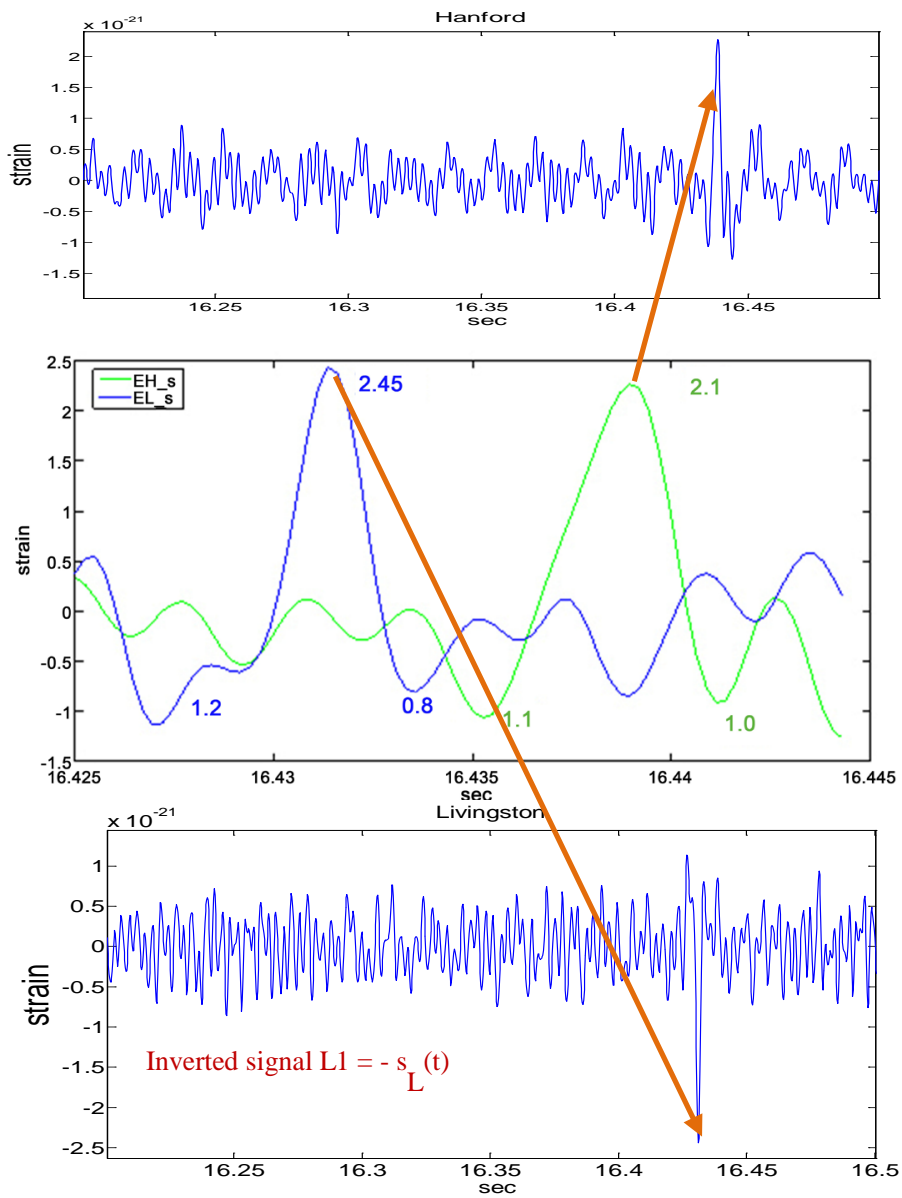

(b)

Figure 4. (a) Recordings of filtered information, the time interval $13.2 \mathrm{sec}-16.8 \mathrm{sec}$. (b) Recordings of filtered information, the time interval $16.2 \mathrm{sec}-16.5 \mathrm{sec}$. Recordings of filtered information, the time interval $16.425 \mathrm{sec}-16.445 \mathrm{sec}$. Regime "Magnifier". For a visual comparison the $\mathrm{L} 1$ data are inverted. 


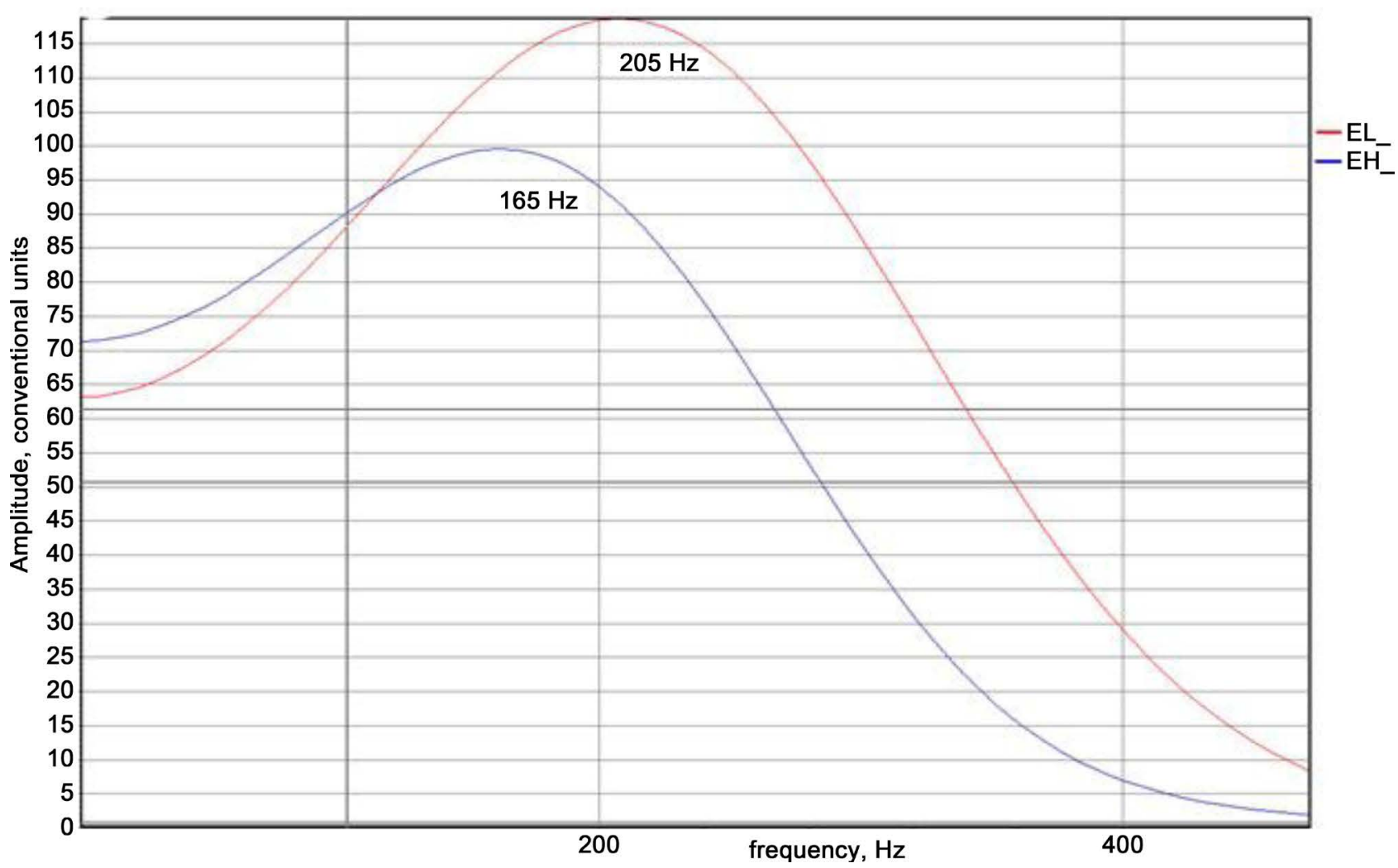

Figure 5. Signal spectra on observatories L1 (red line) and H1 (blue line).

Whitening (rectify) is proposed to conduct in the frequency domain in [5] [6], using the equation:

$$
Y(t)=F^{-1}\left(F_{X}(f) F_{h}(f)\right),
$$

where $F_{X}(f)$-Fourier transform of $X(t)$;

$F_{h}(f)$-transfer function of the whitening filter;

$F^{-1}$-inverse Fourier transform.

The transfer function of the whitening filter is in [5] [6]:

$$
R_{\text {whitening }}(w)=\frac{1}{\sqrt{G_{n}(w)}}, \quad \operatorname{Im}_{\text {whitening }}(w)=0 .
$$

This function has a zero phase.

The filter (Equation (14), Equation (15)) is physical be unrealizable, because any physically realizable filter performs a phase shift [13] [14].

For example, the Butterworth filter is physically realizable (causal) filter.

If the real (true) signals shown in Figure 1, the effect of change in frequency observed well by 0.33 seconds by which you can visually detect signals. With such a difference between the spectra of processes that can be seen before and after 0.33 seconds in Figure 1, obviously the optimum detector would detect these signals (Equation (13)).

However, the detection of the signal is observed on the $0.395 \mathrm{sec}$ on the results of optimal detector (Figure 2, red line) using the input data $h_{L}(t)$ and $h_{H}(t)$. Also according to [5] [6] (February-June 2016) Signal Detection is indicated 
on the 0.39 seconds.

The difference between the times of the signal arrivals indicates a contradiction with the assumption of trueness of image of waveform in Figure 1.

The difference, which is equal to 60 milliseconds, is the result of violation of the principle of causality in the processing of registration data [5] [6].

The reason is that the transfer function (Equation (15)) defines a physically unrealizable (not causal) filter, its impulse response contains non-zero values for $t<0$, and the use of such a filter leads to false detection of signals, shifts the time of their arrival and distortion of their forms (Figure 6) [13] [14].

Chirp signals are not visible after causal filtering (Figure 4, Figure 6), the chirp signals (Figure 1) are false, obtained by the not causal filtering (Equation (14), Equation (15)).

\section{Conclusions}

1) Chirp signals are absent in data LIGO 14.09.2015.

This is established by means of a matched filter and two-stage filtration, therefore signals of the merger of black holes are absent in these data.

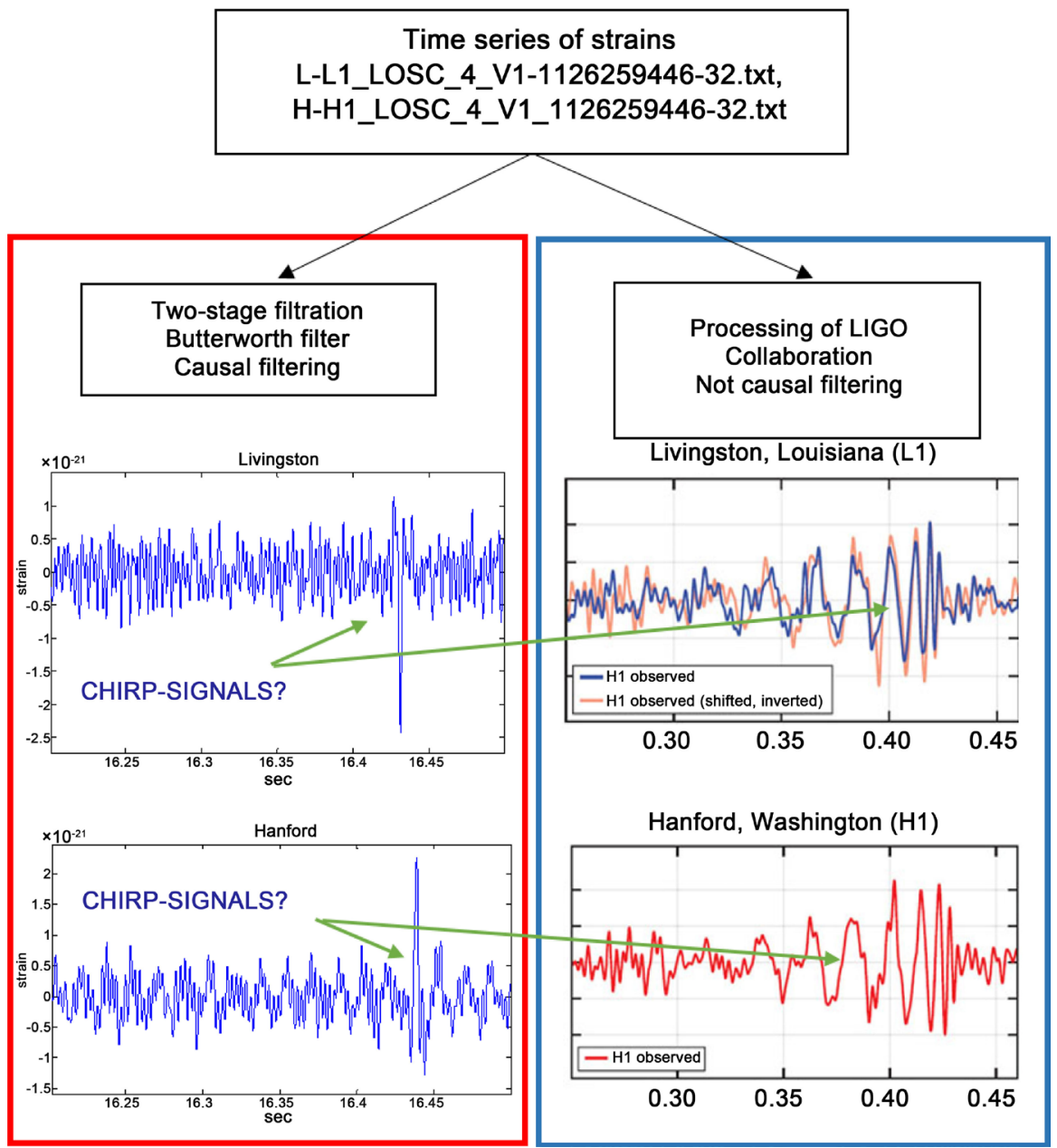

Figure 6. The results of the processing of the same registration data. Left part-causal filtering, the right part-not causal filtering. 
2) Signals of type "soliton" are distinguished by means of bandpass filtering (two steps).

Two pulse signals of different polarity were found in these records.

The corresponding wavelet is: "Mexican hat" or "Sombrero".

3) Optimal detector detects these signals, $\mathrm{SNR} \geq 7$.

4) Signal arrived first at $L 1$ and $\leq 10 \mathrm{~ms}$ later at $H 1$.

5) The signal spectra have a maximum of:

$$
\max S_{H}(f) \approx 165 \mathrm{~Hz}, \max S_{L}(f) \approx 205 \mathrm{~Hz} .
$$

The spectra differ significantly as a result of a violation of invariance of spectra of signals at a filtration (properties №3, 4).

6) It is found that the detected signals at $L 1$ and $H 1$ :

$$
S_{H}(t) \neq-S_{L}(t+\tau)
$$

7) Physical unrealizability (not causal) filter is used to whitening the registration data in [5] [6] and it leads to a false detection signals.

8) It is necessary to be based on the principle of causality when processing signals in physics experiments.

P.S. This article describes a repeatable physical experiment.

Initial data are known: parameters of used Butterworth filters, time intervals of registration and also file names.

Data of registration can be downloaded from website:

https://losc.ligo.org/events/GW150914.

This experiment can be repeated to check the main conclusions.

\section{References}

[1] Einstein, A. (1916) Approximative Integration of the Field Equations of Gravitation, Prussian Academy of Sciences, June 1916. Berlin (Math. Phys.), 688-696.

[2] Hulse, R.A. and Taylor, J.H. (1975) Discovery of a Pulsar in a Binary System. Astrophysical Journal, 195, L51-L53. https://doi.org/10.1086/181708

[3] Taylor, J. H. and Weisberg, J.M. (1982) A New Test of General Relativity- Gravitational Radiation and the Binary Pulsar PSR 1913+16. Astrophysical Journal, 253, 908-920. https://doi.org/10.1086/159690

[4] Weber, J. (1960) Detection and Generation of Gravitational Waves. Physical Review, 117, 306-313. https://doi.org/10.1103/PhysRev.117.306

[5] Abbott, B.P., et al. (2016) Observation of Gravitational Waves from a Binary Black Hole Merger. Physical Review Letters, 116, 061102.

https://doi.org/10.1103/PhysRevLett.116.061102

[6] https://losc.ligo.org/s/events/GW150914/GW150914_tutorial.html

[7] Levin, B.R. (1989) Theoretical Foundations of Statistical Radio Engineering. Radio and Communications, Moscow, $656 \mathrm{p}$.

[8] Repin, V.G. and Tartakovskiy, G.P. (1977) Statistical Synthesis under a Priori Uncertainty and Adapting Information Systems. Sovetskoe Radio, Moscow, 432 p.

[9] Kolmogorov, A.N. (1941) Interpolation and Extrapolation of Stationary Sequences. Izvestiya the Academy of Sciences of the USSR, Ser. Math., No. 5, 3-14.

[10] Wiener, N. (1949) Extrapolation, Interpolation and Smoothing of Stationary Time 
Series. Wiley, NY, 162 p.

[11] Levinson, N. (1947) The Wiener RMS Error Criterion in Filter Design and Prediction. Journal of Mathematical Physics, 25, 261-278.

https://doi.org/10.1002/sapm1946251261

[12] Durbin, J. (1960) The fitting of Time Series Models. Review of the International Statistical Institute, 28, 233-243. https://doi.org/10.2307/1401322

[13] Max, J. (1983) Methods and Signal Processing Appliances at the Physical Measurements. Masson, Moscow, Vol. 1. 312 p.

[14] Lukanenkov, A.V. (2016) What Registered LIGO 14.09.2015? Engineering Physics, No. 8, 64-73.

Submit or recommend next manuscript to SCIRP and we will provide best service for you:

Accepting pre-submission inquiries through Email, Facebook, LinkedIn, Twitter, etc. A wide selection of journals (inclusive of 9 subjects, more than 200 journals)

Providing 24-hour high-quality service

User-friendly online submission system

Fair and swift peer-review system

Efficient typesetting and proofreading procedure

Display of the result of downloads and visits, as well as the number of cited articles

Maximum dissemination of your research work

Submit your manuscript at: http://papersubmission.scirp.org/

Or contact jamp@scirp.org 